\section{Cultura preventiva y condición de salud bucal de pacientes con VIH/SIDA atendidos en un hospital nacional}

\author{
Preventive culture and oral health condition of patients with HIV / AIDS \\ treated in a national hospital
}

\begin{abstract}
Resumen
El objetivo del estudio fue determinar la cultura preventiva y la condición de salud bucal de los pacientes con VIH/SIDA atendidos en un hospital nacional en el ańo 2012. El tipo de estudio fue descriptivo, transversal y retrospectivo. La muestra estuvo conformada por 250 pacientes de ambos sexos. La selección de la muestra fue por conveniencia. El método utilizado para la recolección de datos fue de observación clínica y cuestionario. Los resultados obtenidos fueron: un nivel de cultura preventiva sobre salud bucal bajo, en un 67,6\%; presencia de placa moderada en un 55,6\%; gingivitis moderada en un

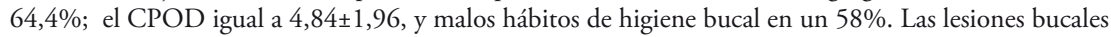
presentadas con mayor frecuencia: candidiasis bucal $(32,8 \%)$ y herpes bucal $(3,2 \%)$. Se logró determinar que los pacientes con VIH/SIDA presentaron mayoritariamente un nivel bajo de cultura preventiva en salud bucal, y un nivel regular de condición de salud bucal.
\end{abstract}

Palabras clave: HIV, prevención de enfermedades, educación para la salud comunitaria, salud bucal, higiene bucal, candidiasis bucal.

\begin{abstract}
The aim of the study was to determine the safety culture and oral health status of patients with HIV / AIDS care in a national hospital in 2012. The type of study was descriptive, cross-sectional and retrospective. The sample consisted of 250 patients of both sexes. The sample selection was convenience. The method used for data collection was questionnaire and clinical observation. The results were: a level of oral health preventive culture low in $67.6 \%$, moderate presence of plaque in $55.6 \%$, moderate in $64.4 \%$ gingivitis; DMFT equal to $4.84 \pm 1.96$, and poor oral hygiene habits by $58 \%$. Oral lesions presented more frequently: oral candidiasis $(32.8 \%)$ and oral $(3.2 \%)$ herpes. It was determined that patients with HIV / AIDS mainly presented a low level of safety culture in oral health, and regular level of oral health status.
\end{abstract}

Keywords: AIDS, disease prevention, community health education, oral health, oral hygiene, oral candidiasis.
Casos Clínicos

\author{
1 Cirujano Dentista, egresada de la Facultad de \\ Odontología de la UNMSM.
}

Correspondencia:

CD. Lisbeth Cynthia Pérez Cahuaya

Dirección: Jr. Los palmitos 488. Urb. Las Violetas. SJL

Correo Electrónico: lipeca25@gmail.com

\section{Introducción}

La infección por el virus de la inmunodeficiencia humana $(\mathrm{VIH})$ constituye un grave problema de salud mundial, porque produce una disminución paulatina de los linfocitos del sistema inmunológico hasta causar su depleción total, provocando múltiples enfermedades, entre ellas las enfermedades bucales, como la Caries dental, Enfermedad periodontal, Candidiasis bucal, Herpes bucal, entre otras, que se han ido incrementando con el paso del tiempo, produciendo un deterioro en la calidad de vida de las personas que la padecen ${ }^{1}$. En el Perú se han registrado más de 20,000 casos de SIDA y más de 30,025 infecciones por $\mathrm{VIH}$, lo que hace un total de 50,025 casos identificados en los últimos ańos. Según recientes estadísticas realizadas, esta enfermedad está relacionada con el estilo de vida, y las costumbres de las personas. De igual manera, las enfermedades bucales son multifactoriales, y no solo se producen cuando existe una alteración entre el huésped, microorganismos, y el sustrato; sino también cuando interactúan factores externos como los malos hábitos de higiene bucal o el bajo nivel de conocimiento sobre prevención de la salud bucal. Por ello, es importante crear estrategias (educación, motivación y promoción de la Salud), para mejorar el nivel de la cultura preventiva en salud bucal, entendiéndose ésta, como la sumatoria de los conocimien-
Fecha de recepción: 22-08-2013

Fecha de aceptación: 27-09-2013 tos preventivos de la salud bucal y los hábitos de higiene bucal, en los pacientes con VIH/SIDA, porque debido a la inmunodeficiencia severa y progresiva que padecen, condiciona la presencia de dichas enfermedades, que afectarían su calidad de vida ${ }^{2}$.

El presente trabajo tuvo como propósito determinar la cultura preventiva y condición de salud bucal de los pacientes con VIH/SIDA atendidos en un Hospital Nacional ya que el odontólogo debe conocer las principales enfermedades bucales que se presentan en esta población vulnerable para que pueda brindarles la atención e información adecuada. 


\section{Material y métodos}

El tipo de estudio fue descriptivo, retrospectivo y transversal. La selección de la muestra fue por conveniencia y estuvo conformada por 250 pacientes de ambos sexos que acudieron al servicio de Infectología del Hospital Nacional Hipólito Unánue durante el periodo de setiembre a diciembre del 2012, con un diagnóstico de VIH/SIDA; entre 18 y 60 ańos, con un tratamiento antirretroviral de un año a más y domiciliados en Lima, Perú. El método utilizado para la recolección de datos fue de observación clínica y un cuestionario, previo consentimiento informado. El cuestionario contenía un total 18 preguntas, divididas en dos secciones, el primero, contenía 12 preguntas sobre conocimientos preventivos de la Salud bucal y la segunda 6 preguntas sobre hábitos de higiene bucal. Cada pregunta presentaba alternativas múltiples, para marcar con un aspa $(\mathrm{X})$. El total obtenido correspondía al nivel de cultura preventiva en salud bucal del paciente. Para determinar la condición de salud bucal, se utilizó una ficha clínica, para medir el índice de CPO-D, el índice gingival de Loe-Silness y el índice de placa bacteriana de Loe-Silness. Se verificaron los resultados según la clasificación del CPOD y de Loe y Silness. Se trabajó con un nivel de confianza de un $95 \%$ y de precisión del $5 \%$. Los datos obtenidos fueron registrados y analizados en el programa estadístico SPSS 17 y para relacionar las variables se utilizó la prueba paramétrica Chi cuadrado. .

\section{Resultados}

La muestra estuvo constituida por 250 pacientes de ambos sexos, con predominio del sexo masculino (62,8 \%). Entre las edades comprendidas la ma- yoría se encontraba en un rango de 29 a 39 ańos de edad (33,2 \%). El 55,2 \% de los pacientes con VIH/SIDA presentaron grado de instrucción secundaria y el 1,2\% no presentaron ningún grado de instrucción (Analfabeto) Tabla 1.

Los pacientes presentaron un nivel de conocimientos preventivos sobre salud bucal bajo y regular, en un $48 \%$ y 37,2 $\%$ respectivamente. (Tabla 2 ). Los hábitos de higiene bucal malos y regulares, en un $58 \%$ y $37,2 \%$ respectivamente. (Tabla 3). Se encontró mayoritariamente un nivel de cultura preventiva en salud bucal bajo en un 67,6\%. (Tabla 4).

Se encontró presencia de placa bacteriana moderada, en un 55,6\%. (Tabla 5). $\mathrm{Y}$ enfermedad gingival moderada en un 64,4\%. (Tabla 6).

Los pacientes con VIH/SIDA presentaron un CPOD entre 2 y 10 piezas dentarias con experiencia de caries con un promedio de 4,84 $\pm 1,96$. (Tabla 7).

Tabla 1. Frecuencia de las variables epidemiológicas de los pacientes con VIH/ SIDA

\begin{tabular}{|c|c|c|c|}
\hline \multicolumn{2}{|c|}{ Variables } & \multirow{2}{*}{$\begin{array}{c}\text { Frecuencia } \\
69\end{array}$} & \multirow{2}{*}{$\begin{array}{c}\text { Porcentaje } \\
27,6 \%\end{array}$} \\
\hline \multirow{4}{*}{ Grupo etario } & $18-28$ & & \\
\hline & $29-39$ & 83 & $33,2 \%$ \\
\hline & $40-50$ & 77 & $30,8 \%$ \\
\hline & $51-60$ & 21 & $8,4 \%$ \\
\hline \multirow{2}{*}{ Sexo } & Femenino & 93 & $37,2 \%$ \\
\hline & Masculino & 157 & $62,8 \%$ \\
\hline \multirow{5}{*}{ Grado de instrucción } & Primaria & 47 & $18,8 \%$ \\
\hline & Secundaria & 138 & $55,2 \%$ \\
\hline & Superior técnico & 37 & $14,8 \%$ \\
\hline & Superior universitario & 25 & $10 \%$ \\
\hline & No presenta (Analfabeto) & 3 & $1,2 \%$ \\
\hline
\end{tabular}

Tabla 2. Nivel de conocimientos preventivos de los pacientes con VIH/ SIDA del Hospital Nacional Hipólito Unánue. Lima-Perú 2012.

\begin{tabular}{ccc}
\hline Nivel de Conocimientos & Frecuencia & Porcentaje \\
\hline Alto & 35 & 14 \\
Regular & 93 & 37,2 \\
Bajo & 122 & 48,8 \\
Total & 250 & 100 \\
\hline
\end{tabular}

Tabla 3. Hábitos de higiene bucal de los pacientes con VIH/SIDA del Hospital Nacional Hipólito Unánue. Lima-Perú 2012.

\begin{tabular}{ccc}
\hline Hábitos de higiene bucal & Frecuencia & Porcentaje \\
Buena & 12 & 4,8 \\
Regular & 93 & 37,2 \\
Mala & 145 & 58 \\
Total & 250 & 100 \\
\hline
\end{tabular}


Tabla 4. Nivel de la cultura preventiva de los pacientes con VIH/ SIDA del Hospital Nacional Hipólito Unánue. Lima-Perú 2012.

\begin{tabular}{ccc}
\hline Cultura preventiva & Frecuencia & Porcentaje \\
\hline Buena & 29 & 11,6 \\
Regular & 52 & 20,8 \\
Mala & 169 & 67,6 \\
TOTAL & 250 & 100 \\
\hline
\end{tabular}

Tabla 5. Presencia de placa bacteriana de los pacientes con VIH/SIDA del Hospital Nacional Hipólito Unánue. Lima-Perú 2012.

\begin{tabular}{ccc}
\hline Índice de placa & Frecuencia & Porcentaje \\
\hline Bajo & 36 & 14,4 \\
Moderado & 139 & 55,6 \\
Alto & 75 & 30 \\
Total & 250 & 100 \\
\hline
\end{tabular}

Tabla 6. Presencia de enfermedad gingival de los pacientes con VIH/SIDA del Hospital Nacional Hipólito Unánue. LimaPerú 2012.

\begin{tabular}{ccc}
\hline Enfermedad gingival & Frecuencia & Porcentaje \\
\hline No presenta inflamación & 4 & 1,6 \\
Inflamación leve & 33 & 13,2 \\
Inflamación moderada & 161 & 64,4 \\
Inflamación severa & 52 & 20,8 \\
Total & 250 & 100 \\
\hline
\end{tabular}

Tabla 7. CPO-D de los pacientes con VIH/SIDA del Hospital Nacional Hipólito Unánue. Lima-Perú 2012.

\begin{tabular}{cccccc}
\hline Variable & Número de casos & ÍNDICE DE CPOD & Desviación típica & Valor Mínimo & Valor máximo \\
\hline CPOD & 250 & 4,84 & 1,96 & 2 & 10 \\
\hline
\end{tabular}

\section{Discusión}

El presente trabajo determinó la cultura preventiva y condición de salud bucal en 250 pacientes con VIH/SIDA, de los cuales el $62,8 \%$ pertenecían al sexo masculino y el $37,2 \%$ al sexo femenino, estos resultados fueron similares a estudios publicados por Garibay L y $\mathrm{Col}^{3}$ en el cual encontraron que de una muestra de 128 pacientes infectados por VIH, 74 pacientes pertenecían al género masculino y 57 al género femenino. En cuanto al rango de edad en la presente investigación se encontró que el grupo etario predominante fue entre 29-39 ańos de edad (33,2\%), lo cual se asemeja a los resultados de estudios publicados por otros autores tales como Tovar $\mathrm{V}$ y Col. ${ }^{4}$ en donde se encontró que la edad promedio de los pacientes infectados osciló entre los 26 y 35 años de edad. Este estudio muestra que el $55,2 \%$ de los pacientes con VIH/SIDA presentaron grado de instrucción secundaria completa, resultado que contrasta con el obtenido por Marcus M y col. ${ }^{5}$ quien reportó que los pacientes con VIH/SIDA se encuentran en bajo nivel educativo y grado de instrucción primaria.

Con respecto a la cultura preventiva (Nivel de conocimiento sobre salud bucal y hábitos de higiene bucal) de los pacientes con VIH/SIDA, son escasos los trabajos realizados, sin embargo en este estudio se evidenció que el 67,6 $\%$ de los casos estudiados presentaron un nivel bajo de Cultura preventiva, en donde se observó que el 48,8\% del total presentaron un bajo Nivel de conocimientos preventivos sobre Salud bucal y el $58 \%$ del total presentaron malos hábitos de higiene bucal, estos resultados fueron similares a los estudios realizados por diferentes autores tales como Otero ${ }^{6}$, quien determinó un bajo nivel de conocimientos sobre salud bucodental y que estaban relacionados con la baja motivación por asistir al estomatólogo $(86.7 \%)$, actitud negativa ante el hábito de fumar (70 \%) y la baja percepción de la relación entre la salud bucal y la salud general.

La condición de salud bucal que se observó en los pacientes con VIH/ SIDA en este estudio fue regular, siendo el índice de CPOD 4,84 $\pm 1,97$, presencia de 2 a más dientes afectados por caries dental, el $64.4 \%$ presentó gingivitis moderada y en el $55.6 \%$ se evidenció placa bacteriana moderada, estos resultados fueron similares a los estudios realizados por otros autores tales como Dávila $\mathrm{M}$ y col. ${ }^{7}$ quienes determinaron, durante un examen bucal, que el $66 \%$ de los pacientes con VIH/SIDA sufren Caries dental. Santana $\mathrm{Y}$ y col. ${ }^{8}$, quienes determinaron que el $58.9 \%$ de los pacientes con VIH/ SIDA presentaron 5 a más dientes afectados por caries dental, el $46.1 \%$ de 
los pacientes presentó placa bacteriana moderada, siendo el cálculo dental, la condición periodontal más prevalente, en el $49.2 \%$ de los casos y el $77.2 \%$ de los pacientes requerían tratamiento periodontal. Por otra parte, en este estudio se observó Candidiasis bucal en un $32,8 \%$ de los casos y herpes oral en un 3, $2 \%$, estos resultados fueron similares a los estudios publicados por diferentes autores, tales como Medina NE y Col.; Arteaga FJ y Col; Estrada $\mathrm{JH}$ y Col', 10,11, quienes estudiaron las manifestaciones bucales y analizaron infecciones oportunistas prevaleciendo lesiones como candidiasis bucal $(12,8$ $\%)$, herpes bucal simple $(4,4 \%)$ en los pacientes con VIH/SIDA examinados.

\section{Conclusiones}

Los pacientes con VIH/SIDA que acudieron al servicio de Infectología del Hospital Nacional Hipólito UnánueMINSA presentaron un nivel de cultura preventiva en salud bucal bajo y una condición de salud bucal regular. La deficiente condición de salud bucal estuvo influenciada por los malos hábitos de higiene bucal y el bajo Nivel de conocimientos sobre Salud bucal. Pacientes con VIH/SIDA con malos hábitos de higiene bucal presentaron acumulación de placa e inflamación gingival moderada debido a que sólo se cepillaban dos veces al día y nunca utilizaron seda dental y enjuagatorio bucal, asimismo por una falta de información sobre salud bucal. La mayoría de los pacientes presentaron alto riesgo de caries dental, el cual estuvo influenciado por el hábito de consumir chicles, caramelos o chocolates más de una vez al día. Existe prevalencia de la Candidiasis bucal sobre el herpes bucal en los pacientes con VIH/SIDA y estos resultados fueron similares a los reportados por la Literatura. Los pacientes con VIH/ SIDA conocen poco sobre prevención y promoción de la Salud bucal, debido a la falta de información que reciben por parte de los profesionales de la Salud, lo que influyó en el nivel bajo de la cultura preventiva que presentaron dichos pacientes. La cultura preventiva estuvo influenciada con el grado de instrucción que presentaron los pacientes; el sexo y grupo etario no influenciaron en ella. Existe una relación significativa entre la cultura preventiva y la condición de Salud oral, según el índice de placa, índice gingival e índice de CPOD.

\section{Referencias Bibliográficas}

1. Reznik S. Topics in HIV Medicine, Perspective - Oral Manifestations. International AIDS Society-USA 2006;13 (5):143-8.

2. Cuenca E. Odontología Preventiva y Comunitaria. Principios, métodos y aplicaciones, $5^{\circ}$ Edición. Barcelona: Editorial Masson S.A.; 2002. 143-145 p.

3. Garibay L, Cisneros L. Manifestaciones estomatológicas en pacientes VIH/SIDA. Odontolo. Sanmarquina 2007;10(1):39-40.

4. Tovar V, Guerra M, Bravo I, Albornoz E, Lambertini A, Ibarra $G$ et al. Manifestaciones Bucales e Infecciones oportunistas más frecuentes encontradas en 208 pacientes con infección por VIH/SIDA. Acta odontol. Venez. 2002;40 (3):3-8.

5. Marcus M, Maida CA, Coulter ID, Freed JR. Un análisis longitudinal de la necesidad no satisfecha de tratamiento oral en una muestra nacional médica de pacientes VIH. Am J Public Health. 2005;95(1):73-5.

A Longitudinal Analysis of Unmet Need for Oral Treatment in a $\mathrm{Na}$ tional Sample of Medical HIV Patients
6. Otero J. Conocimientos, actitudes y creencias relacionadas con la salud bucal en personas que viven con VIH/SIDA. Portales médicos. 2007; 9(3):12-5.

7. Dávila ME, Tagliaferro Z, Parra H, Ugel E. Diagnóstico Bucal de las personas que viven con VIH/ SIDA. Rev Od Los Andes. 2009; 4(2):13-20.

8. Santana Y, Navas R, Morón A, Chaparro N, García R, Suárez I. Condiciones de salud-enfermedad bucal en pacientes adultos VIH/SIDA. Acta odontol. Venez. 2008;46(2):1-7.

9. Medina NE, Brett MM, Betancourt FA, Patiño JC. Frecuencia de lesiones en cavidad oral de pacientes con pacientes con VIH/ SIDA en el Hospital universitario San Ignacio de Bogotá, Colombia (2005-2010). Revista Javeriana. 2012;31(66):51-58.

10. Arteaga FJ, Quiñones B, Prado JR. Manifestaciones periodontales de la infección por el virus de inmunodeficiencia humana adquirida. Rev Od Los Andes. 2008;3(1):54-9.

11. Estrada JH. Lesiones orales asociadas con la infección por VIH/ SIDA en individuos sin tratamiento antirretroviral en el Hospital San Juan de Dios Bogotá. Biomedica. 2001; 2(1): 333-44.

\section{Agradecimiento}

Agradecemos al Vicerrectorado de Investigación de la UNMSM por el apoyo económico para la ejecución de este estudio 